Apidologie, 1976, 7 (2), 151-168.

\title{
UNTERSUCHUNGEN DER AUSFLUGSAKTIVITÄT VON JUNGEN BIENENKÖNIGINNEN UNTER FESTLANDS- UND INSELBEDINGUNGEN MITTELS LICHTELEKTRONISCHER ÜBERWACHUNG
}

\author{
Recherches sur l'activité de vol de jeunes reines d'abeille \\ dans des conditions continentales et insulaires, \\ à l'aide d'un dispositif photoélectronique
}

Bernhard VERBEEK

Aus dem Biologie-Seminar der PH Ruhr, Abteilung Hagen

Stirnband 10,D 58 Hagen

(Seminardirektor Professor Dr. Christian Ullrich)

\section{SUMMARY}

\author{
INVESTIGATION OF THE FLIGHT ACTIVITY OF YOUNG \\ HONEYBEE QUEENS UNDER CONTINENTAL AND INSULAR \\ CONDITIONS BY MEANS OF PHOTOELECTRONIC CONTROL
}

Investigations made in the town of HAGEN (border of Sauerland, mountains of middle altitude, West Germany) and on the Isle of Juist (North Sea) showed by means of photoelectronics, reacting selectively on marked queens that there exist characteristic distinctions between continent and island.

On the islands, the queens' flights were more frequent and shorter. The altitude of the drones amounts only a few meters. Attempts of copulation - mostly ineffective - take place at a very low altitude; at captive flight experiments already at $1,5 \mathrm{~m}$.

On the continent, the flights were rather infrequent, mostly of longer duration and, first of all, higher.

A second series of experiments on the Isle of Jurs,, where there were - opposed to the first series - no drones in the mating station, but a colony with drones $5 \mathrm{~km}$ away, brought very good mating results.

Possible causes and biological meaning of the observed behaviour patterns are discussed. 


\section{ZUSAMMENFASSUNG}

Bei Versuchen in Hagen (Mittelgebirge, Rand des Sauerlandes) und auf der Nordseeinsel Juist zeigte sich unter Einsatz einer selektiv auf markierte Königinnen ansprechenden Lichtelektronik, daß bezüglich der Paarungsaktivität deutliche Unterschiede zwischen Festland und Insel auftreten.

Auf den Inseln fliegen die Königinnen häufiger und kürzer aus. Die Flughöhe der Drohnen beträgt nur wenige Meter. Paarungsversuche — meist erfolglos — finden schon in sehr geringer Höhe statt, im Fesselflugversuch schon in einer Höhe von $1,5 \mathrm{~m}$.

Die Ausflüge auf dem Festland waren seltener, meist länger und vor allem höher.

Eine zweite Inselserie bei der im Gegensatz zur ersten keine Drohnen mehr auf der Belegstelle waren, dafür aber ein Volk mit Drohnen $5 \mathrm{~km}$ entfernt stand, brachte - anders als im ersten Versuch - sehr gute Begattungsergebnisse. diskutiert.

Mögliche Ursachen und biologischer Sinn der beobachteten Verhaltensweisen werden

\section{1. - EINFÜHRUNG}

Das langlebige Dogma, eine Bienenkönigin würde nur ein einziges Mal von einem einzigen Drohn begattet, wurde zunächst von RoBerts (1944, zit. nach Büdel-Herold), dann von Alber, Jordan und F. und H. Ruttner (1955) umgestoßen, und zwar aufgrund von direkten Beobachtungen und von Nachkommenschaftsprüfungen. Seither hat sich eine Reihe weiterer Autoren mit dem Problem der Paarungsflüge befaßt. Es wurde vor allem einwandfrei nachgewiesen, da $\beta$ auf einem einzigen erfolgreichen Paarungsfluge in der Regel mehrere Drohnen begatten (Mehrfachbegattung im Gegensatz zur wiederholten Begattung auf verschiedenen Ausflügen). TrJasko (1956) wies für einen Flug 16-17 Drohnen nach, 17 Drohnen gibt auch Woyke (1960, zit. nach Zander-Weiss) als beobachtetes Maximum an. Das Mittel liegt natürlich niedriger. Einfachbegattungen sind selten. Mit dem speziellen Problem des Königinnenfluges auf Nordseeinseln befaßten sich bisher Drescher, Meinen und Englert. Das Verhalten der Geschlechtstiere auf einer Insel weicht in einigen Punkten erheblich von dem auf dem Festland gezeigten ab.

\section{2. - METHODE UND MATERIAL}

Da lückenlose direkte Beobachtungen über einen längeren Zeitraum praktisch nicht möglich sind, wurde bisher in der Regel den Königinnen der Ausflug durch ein Gitter versperrt, welches vom Untersucher geöffnet werden mußte, wenn sich die Königin dort zeigte. Dieses Verfahren schließt aber nicht aus, daß die Königinnen in ihrem Verhalten beeinflußt werden. Aus diesem Grunde wurde in der vorliegenden Arbeit eine zu diesem Zweck entwickelte Apparatur eingesetzt, die photoelektronisch ausgelöst, ein akustisches und optisches Signal gibt, sobald die Königin eine bestimmte Stelle in dem zum Flugloch führenden $20 \mathrm{~cm}$ langen Kanal passiert. Die Anlage (Abb. 1) spricht nur auf markierte Bienen an, in unserem Falle eben auf die Königin. Die beim Aus- und Eingehen ausgelösten Stromstöße setzen eine akustische und optische Signalanlage in Tätigkeit und werden außerdem auf einem Zeitmarkenschreiber registriert. So erhält man außer dem Beobachtungsprotokoll noch ein Zeitmarkenprotokoll. Da 


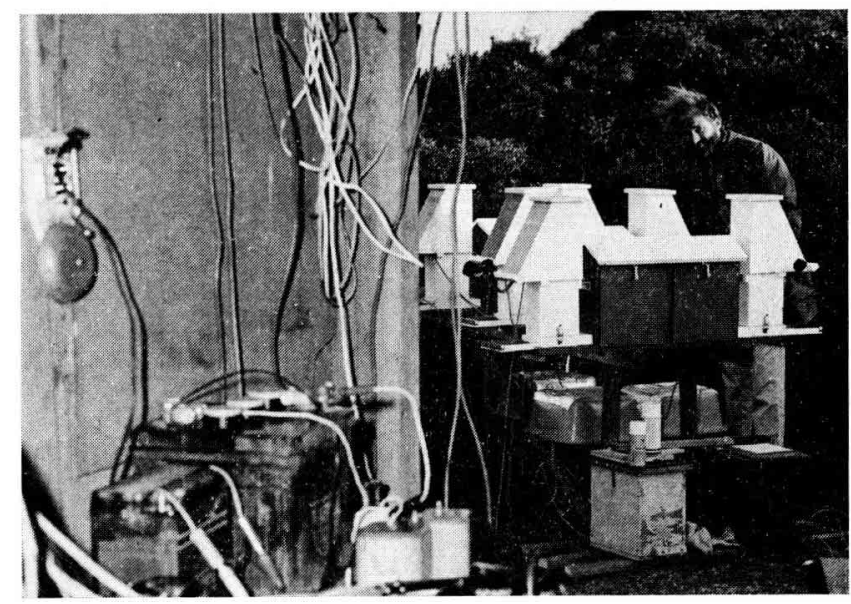

Авв. 1. - Die auf der Insel Juist aufgestelle Apparatur (Foto Gröl).

FIG. 1. - Appareillage installé sur l'île de Juist (Photo Gröl).

zu bestimmten Zeiten mehrere Königinnen gleichzeitig flogen und deshalb manchmal die Beobachtungsprotokolle bezüglich der Zeitangaben lückenhaft wurden, erwies sich der Zeitmarkenschreiber als wichtige Hilfe. Zeitmarken allein besagen jedoch nicht unbedingt, daß tatsächlich ein Ausflug stattgefunden hat. Es könnte die Königin am Flugloch oder schon an der " Passierstelle ” wieder umgekehrt sein, was tatsächlich öfters vorkam. Schließlich gibt auch nur die direkte Beobachtung Auskunft über ein eventuell vorhandenes Begattungsmal.

Dieses Gerät, das schon genauer beschrieben wurde (B. und R. VERBEEK 1974), liefert also nicht automatisch vollständige Beobachtungsprotokolle. Sein großer Vorteil gegenüber den bisherigen Verfahren liegt nur darin, daß die Königinnen nicht durch ein Gitter behindert werden, daß sie vielmehr jederzeit sofort den Versuchskasten verlassen können und dennoch der Aufmerksamkeit des Beobachters nicht entgehen.

Als Versuchsmaterial dienten ausschließlich Carnica-Königinnen, die wir entweder von der Landwirtschaftskammer Münster oder von der Universität Bonn bezogen. Sie wurden in üblicher Weise in verglasten Einwabenkästchen untergebracht. Zur Beobachtung konnten die wärmedämmenden Seitenwände der Schutzhäuschen entfernt werden.

\section{3. - BEOBACHTUNGEN UND ERGEBNISSE}

\section{3.-1. Versuche auf dem Festland}

Erste Festlandserie. Vier Königinnen aus Münster wurden am 27. 6. 1973 in Hagen eingesetzt; ihr Schlüpftermin war der 25. 6. Das Wetter war warm und windarm. Am 4. Lebenstag der Versuchsköniginnen herrschten Temperaturen zwischen 18 und $31{ }^{\circ} \mathrm{C}$ (gemessen am Stand), nach vormittäglichem Sonnenschein setzte gegen 14, 15 Uhr heftiger Regen ein. 


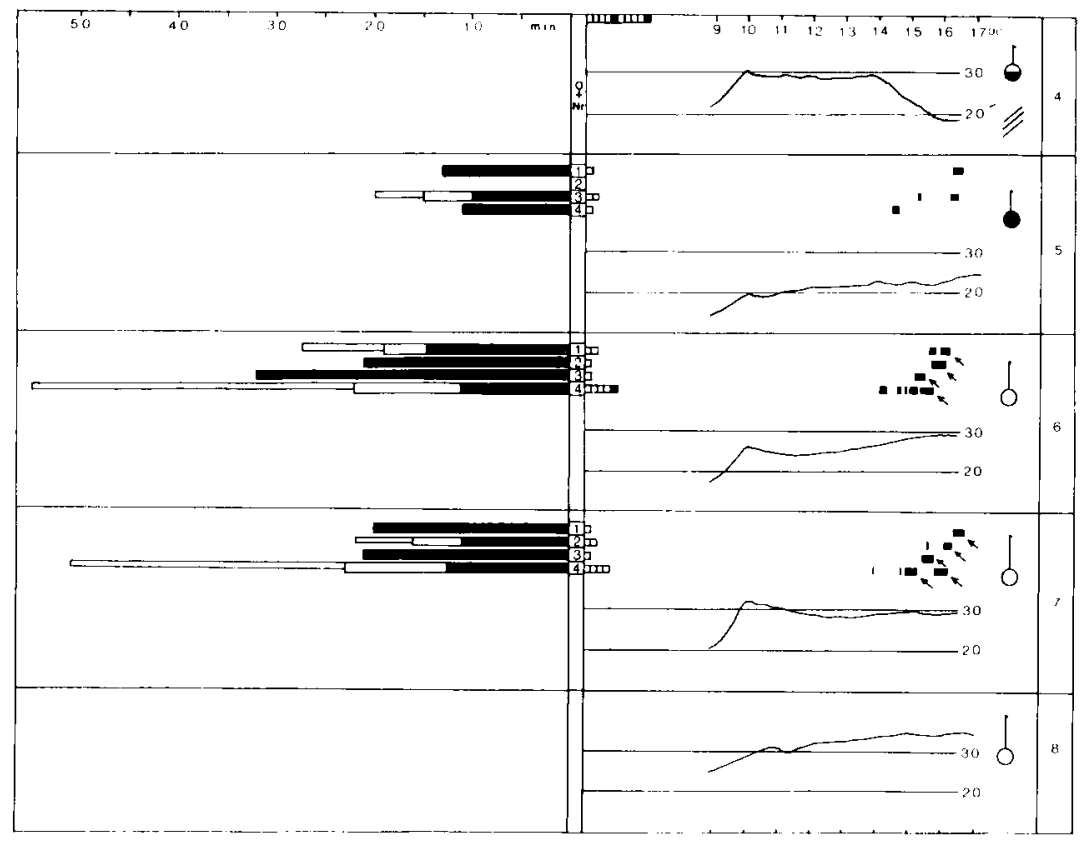

Авв. 2a. - Graphische Darstellung der Ergebnisse des 1. Festlandsversuchs (4. Lebenstag - 28.6.1973).

Fig. 2a. - Représentation graphique des résultats de la première expêrience faite sur le continent (4e jour de vie 28.6.1973.)

Mittelspalte : Die Nummern der einzelnen Königinnen.

Rechte Seite : Die Flugaktivität der einzelnen Königinnen im Laufe des jeweiligen Tages. Pfeile: Begattungsmal. Darunter der Verlauf der Temperaturkurve. Die Kästchen neben der Mittellinie symbolisieren die Anzahl der jeweiligen Ausflüge. Schwarze Kreise : Himmel bedeckt. Weiße Kreise : Himmel wolkenlos. Lange Fähnchen : Grundgeschwindigkeit (je Schrägstrich $1 \mathrm{~m} / \mathrm{sec}$ ). Kurze Fähnchen : maximale Windgeschwindigkeit. Schraffur : Regen.

Linke Seite : Schwarze Säulen : Mittlere Flugdauer der jeweiligen Königin am jeweiligen Tag. Weiße Säulen (breit) : Dauer des längsten Ausfluges. Weiße Säulen (schmal) : Summe der Flugminuten am jeweiligen Tag.

Colonne médiane : numéro des reines.

Partie droite : activité journalière de vol de chaque reine. Les traits indiquent un accouplement. Dessous figure la courbe de température. Les petites cases à côté de la ligne médiane symbolisent le nombre de vols journaliers. Les cercles noirs représentent un ciel couvert, les cercles blancs un ciel dégagé. Les longs fanions indiquent la vitesse au sol (chaque trait oblique $=1 \mathrm{~m} / \mathrm{sec}$ ), les fanions courts la vitesse maximale du vent, les hachures de la pluie.

Partie gauche : colonnes noires : durée moyenne de vol de chaque reine chaque jour. Colonnes blanches (larges) : durée du vol le plus long. Colonnes blanches (étroites) : total journalier des minutes de vol. 


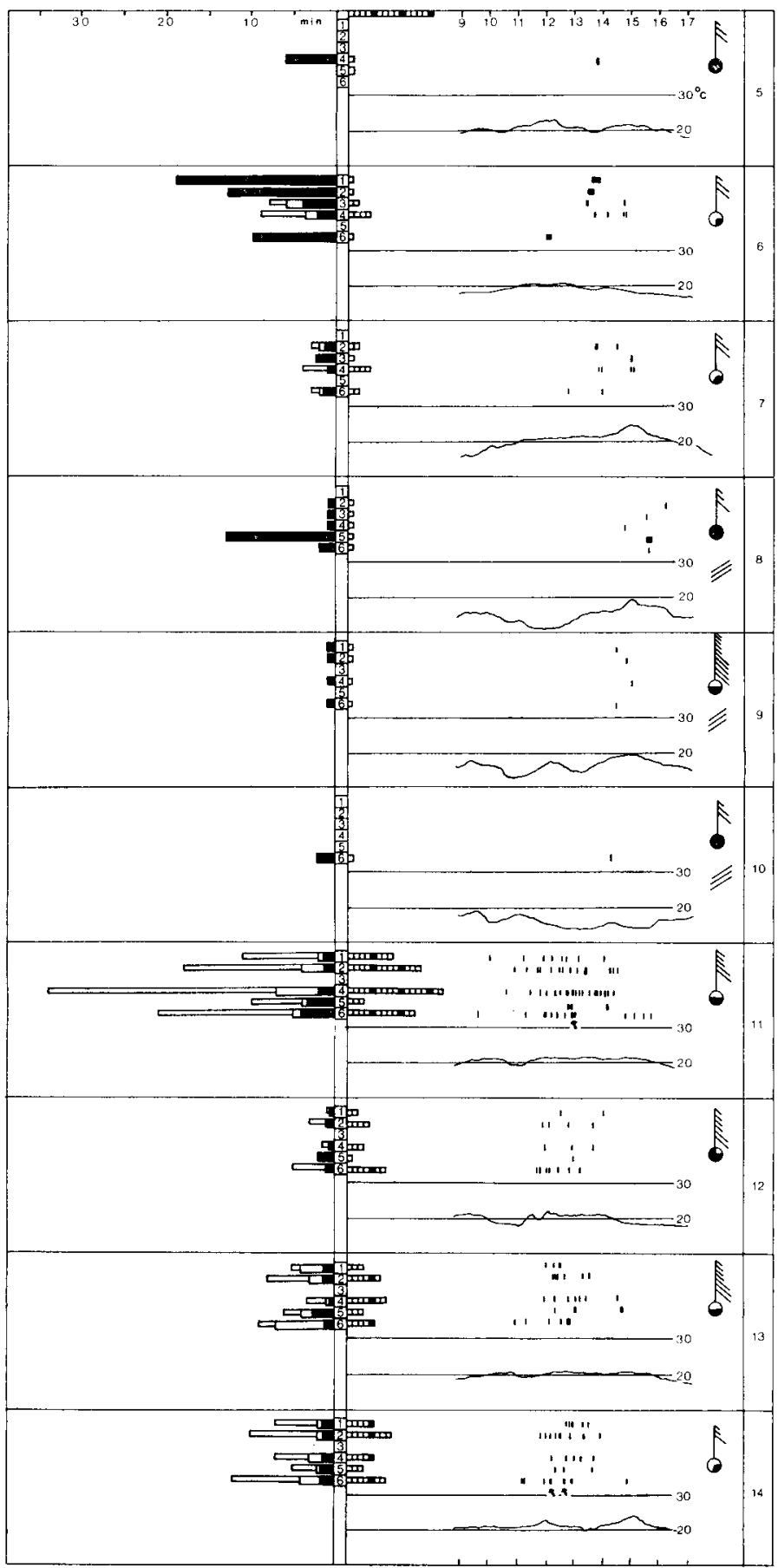

AвB. 2b. - 1. Inselversuch (5. Lebenstag = 21.7.1974).

FIG. 2b. - Première expérience sur l'île (5e jour de vie $=21.7 .1974)$. 
Erster Ausflug. Am 5. Lebenstag (höchste Temperatur 24, ${ }^{\circ}$ ) schien die Sonne am frühen Nachmittag nicht, es herrschte vielmehr eine ganz ungewöhnlich geringe Lichtintensität. Dennoch verließ die erste Königin (vgl. Abb. 2a $\mathrm{Nr}$. 4) zwischen 14,25 Uhr und 14,36 Uhr ihren Stock und flog in der näheren Umgebung herum, landete zwischendurch auf der Apparatur, auch einmal ca. $3 \mathrm{~m}$ entfernt auf dem Rasen, schlüpfte schließlich nach längerem Schweben vor dem Flugloch wieder ein. Bis 16,30 $\mathrm{Uhr}$ haben noch 2 weitere der vier im Versuch befindlichen Königinnen ähnliche bis ca. $40 \mathrm{~m}$ entfernte Orientierungsflüge unternommen. Eine Königin flog zweimal aus und interessierte sich beide Male auffällig für das Flugloch der Nachbarin, wo sie von Bienen angegriffen wurde.

Paarungsflüge. Am 6. Lebenstag (30. Juni) begann die Flugaktivität bei zwei Königinnen wieder mit Orientierungsflügen in geringer Entfernung vom Stock (vgl. graphische Darstellung in Abb. 2a). Zu den tatsächlichen Hochzeitsflügen, deren Erfolg am Begattungsmal sichtbar wurde, entfernten sich die Königinnen rasch hochkreisend außer Sichtweite. Um 16, 12 Uhr war die letzte der 4 Königinnen begattet. Der Tag war sonnig bei einer Höchsttemperatur von $28^{\circ} \mathrm{C}$. Am 7. Tag (Sonne, Temperaturmaximum $34^{\circ} \mathrm{C}$ ) wurden 3 der Königinnen auf einem, die 4. sogar auf zwei Flügen nachweislich begattet. Somit hatte jede Königin 2 bis 3 Begattungsflüge hinter sich und wahrscheinlich erheblich mehr Begattungen.

Eiablage. Obgleich das Thermometer am nächsten Tage bei sonnigem Wetter auf $35{ }^{\circ} \mathrm{C}$ anstieg und auch die folgende Woche eine ausgesprochen sonnige Schönwetterperiode war, fanden keine Flüge mehr statt. Zwei Königinnen gingen am 9. Lebenstag in Eiablage, eine am 11., die 4. (auf 3 Flügen begattete) wurde am 10. Tag tot herausgeschleppt.

Zweite Festlandserie. Versuche, die àm 5. 6. 74 (Schlüpftermin) in Hagen wieder mit Königinnen aus Münster begannen, fielen in eine weniger günstige Wetterperiode. Am 2. Lebenstag betrug die höchste Tagestemperatur $18^{0}$, dann stieg sie bis zum 7. Tag nicht über die 160-Marke. Am 8. Tag stieg die Temperatur bis auf knapp $19^{\circ} \mathrm{C}$. Vier der fünf eingesetzten Königinnen benutzten diesen Temperaturanstieg trotz bedecktem Himmel für Orientierungsflüge in Stocknähe bis zu 7 Minuten Dauer oder zeigten sich wenigstens zu Fuß am Flugloch. Nachdem am 9. Tag (Höchsttemperatur 210, meist stark bedeckt) wieder nur Flüge in der näheren Umgebung unternommen worden waren, fanden am 10. Tag bei vorwiegend sonnigem Wetter und Temperaturen zwischen 22 und $27^{\circ} \mathrm{G}$ auch Begattungsflüge statt. Drei Königinnen kehrten nach einem, eine nach 4 (!) Flügen mit einem Begattungszeichen zurück. Letztere und die bisher noch nicht nachweislich begattete Königin flogen am 11. Tag (Höchsttemperatur $28{ }^{\circ} \mathrm{C}$ ) noch einmal mit Erfolg aus.

Wirkung von Absperrgittern. Bei zweien der mindestens einmal begatteten 
Königinnen testete ich an diesem Tage die Wirkung von Absperrgittern. Eine versuchte etwa eine halbe Stunde ins Freie zu kommen. Diese Ausflugsabsicht wäre bei der herkömmlichen Absperrgittertechnik sicherlich nicht unbemerkt geblieben. Die zweite aber ging sofort wieder in den Stock zurück, ohne einen weiteren Versuch zu unternehmen.

Eiablage. Obgleich die nächsten Tage recht warm waren, fanden keine Flüge mehr statt, vielmehr begann die Königin, die sich am Vortage sofort mit dem Absperrgitter abgefunden hatte, am 12. Lebenstag zu legen, am 13. folgten die auf 5 Flügen begattete Königin und die, die sich so lange vor dem Absperrgitter aufgehalten hatte. Erst am 16. ging die nächste und am 17. Tag die letzte in Eiablage. Diese Königin war auch als letzte ausgeflogen und begattet worden (1 Begattungsmal) und hatte entschieden das schwächste EWKVölkchen, während die Königin, die 5 Begattungszeichen heimtrug, im stärksten Völkchen war.

Drohnenmangel. Im Juni 1973 (1. Festlandserie) fanden die Königinnenflüge bei gleichbleibend gutem Wetter an höchstens 3 Tagen zwischen dem 5 . und 7. Lebenstag statt. Die 2. Festlandserie im Juni 1974 bestätigte, daß schlechtes Wetter die Flüge verschieben kann (8. bis 11. Lebenstag). Daß aber auch noch andere Faktoren, nämlich Drohnenmangel und die Ausdifferenziertheit der Königinnen, einen Einfluß auf die Flugaktivität haben können, macht ein “ Nebenversuch » im September 1973 wahrscheinlich, der mit zwei Königinnen verschiedener Größe durchgeführt wurde. Die größere flog bei durchgehend günstigerem Wetter vom 4 . bis zum 14 . Lebenstag. Am Abend des 8. Tages war sie immerhin auf mindestens zwei Flügen begattet, flog aber noch ohne Begattungszeichen bis zum 13. Tag, vielleicht wegen des infolge Drohnenmangels geringen Füllungsgrades der Samenblase (WOY KE 1964, 1966) und ging am 16. Tag in Eiablage. Dagegen flog die kleinere erstmalig am 9. und zeigte erst am 14. Lebenstag ein Begattungsmal. Danach flog sie nicht mehr aus (kleine Samenblase ?). Erst am 21. Lebenstag begann sie mit der Eiablage. Die Gesamtflugdauer dieser Königinnen war ungewöhnlich lang (vgl. Tabelle 1).

\section{3.-2. Versuche auf einer Insel}

Erste Inselserie. Ungünstiges Wetter, kurze Flüge. Einige Überraschung brachten die Versuche vom 19.7. bis 17. 8. 1974 auf Juist. Es wurde zunächst wieder Material aus Münster verwendet (Schlüpftermin 17. 7.). Trotz relativ gutem Wetter flogen die Königinnen am 7. Lebenstag kaum, nachdem sie sich am Vortage mit einer Ausnahme ausgiehig in der näheren Umgebung orientiert hatten (vgl. Abb. 2b). Das Wetter wurde an den folgenden Tagen zunehmend schlechter, bis am 11. Lebenstag bei keineswegs optimalem Flugwetter (Höchsttemperatur $22^{\circ} \mathrm{C}, 2-5 \mathrm{~m} / \mathrm{sec}$ Windgeschwindigkeit dicht über 
B. VERBEEK

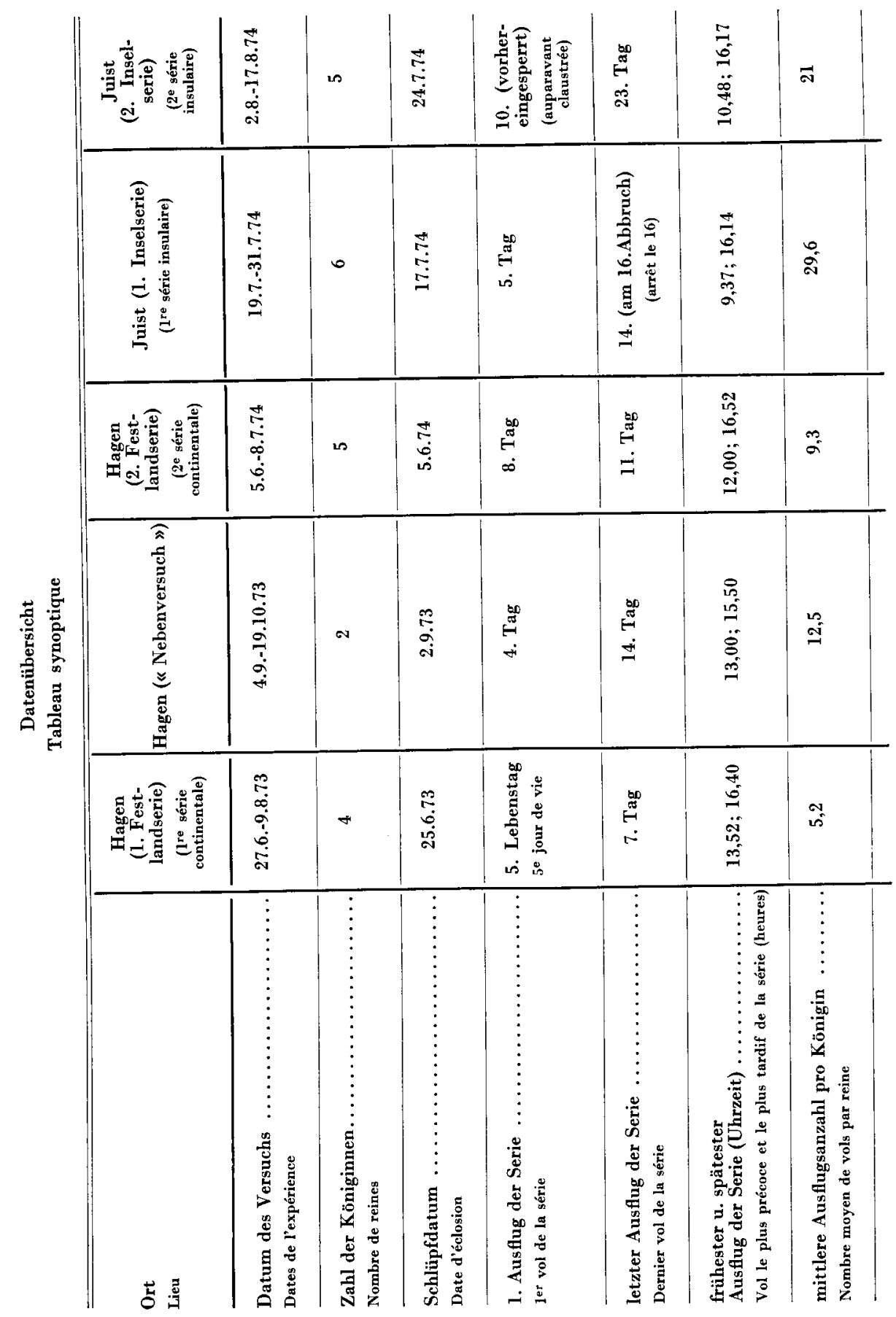




\begin{tabular}{|c|c|c|c|c|c|c|c|}
\hline 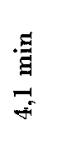 & $\begin{array}{l}\stackrel{\Xi}{\sharp} \\
\infty \\
\infty \\
\wedge\end{array}$ & 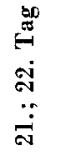 & 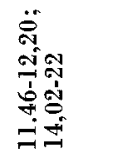 & $\stackrel{m}{i}$ & $\begin{array}{l}\stackrel{E}{G} \\
\stackrel{\Xi}{m} \\
i\end{array}$ & 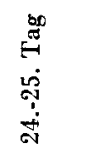 & \\
\hline 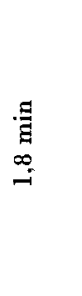 & 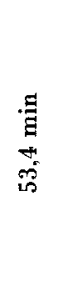 & 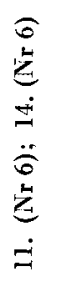 & 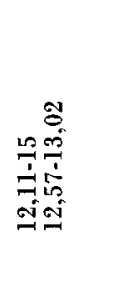 & 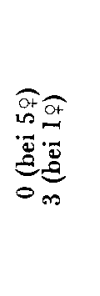 & $\begin{array}{l}\tilde{z} \\
\dot{g} \\
p \\
\dot{n}\end{array}$ & 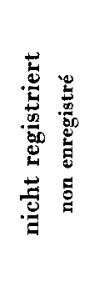 & 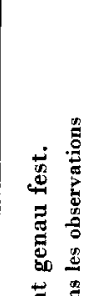 \\
\hline 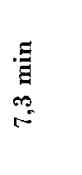 & $\begin{array}{l}\vec{\Xi} \\
\stackrel{g}{\Xi} \\
\wedge\end{array}$ & 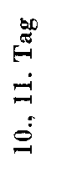 & 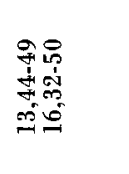 & 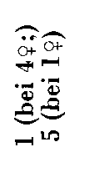 & 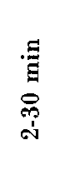 & 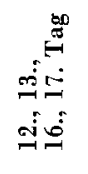 & 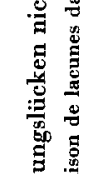 \\
\hline 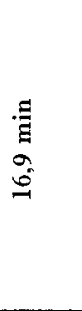 & $\begin{array}{l}\vec{\Xi} \\
\vec{\Xi} \\
\bar{\Delta}\end{array}$ & 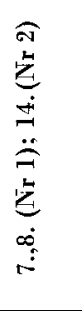 & 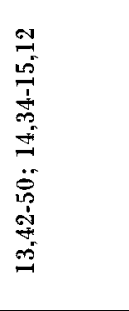 & $\stackrel{9}{I}$ & 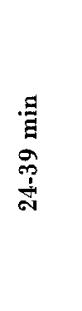 & 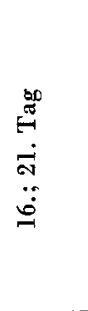 & 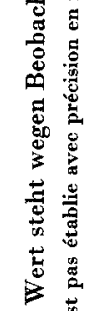 \\
\hline 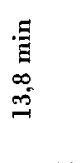 & 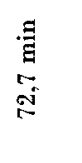 & 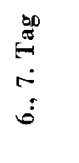 & 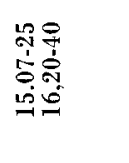 & $\stackrel{\infty}{\sim}$ & 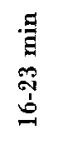 & 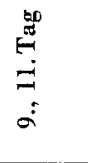 & 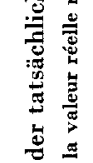 \\
\hline 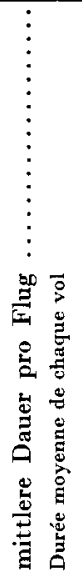 & 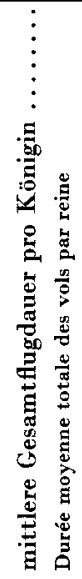 & 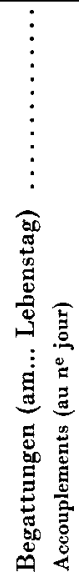 & 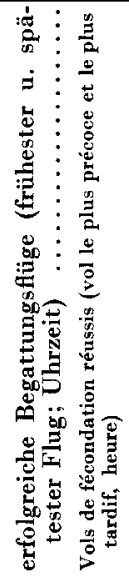 & 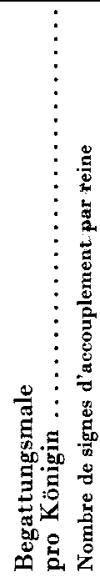 & 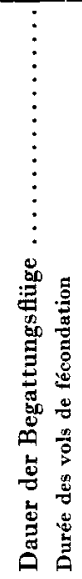 & 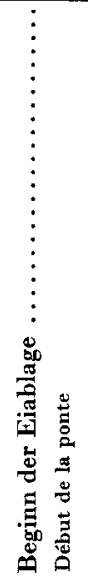 & 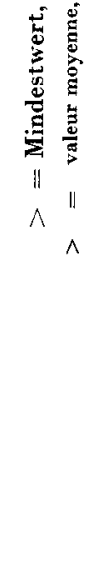 \\
\hline
\end{tabular}


der Strauchvegetation) eine rege Flugaktivität einsetzte (Abb. 2b). (Dieses auch in zwei anderen Serien beobachtete « Nachholbedürfnis » entspricht nicht ganz den Beobachtungen Englerts, denen zufolge nach einem guten Flugtag auch bei schlechterem Wetter noch Flüge folgen können, während die Königinnen nach schlechten Flugtagen erst bei Temperaturen über $20^{\circ} \mathrm{C}$ ausfliegen. H. Ruttene (1972) berichtet für Drohnen von besten Fangergebnissen auf Drohnensammelplätzen nach einer Schlechtwetterperiode. Diese Beobachtung an Drohnen und meine an Königinnen entsprechen dem Phänomen der Reizschwellenerniedrigung, dem zufolge sich Triebe aufstauen und dann bei geringer Reizstärke heftiger entladen können.) Die höchste Ausflugszahl bei einem Tier ( $($ N Nr. 4 dieser Serie) betrug bis zum 14. Lebenstag 43. Die Einzelflüge waren meist sehr kurz (Abb. 2b). Am 15. Tag war schlechtes Wetter, am 16. wurde diese Serie abgebaut, um für die nächste Platz zu schaffen. Da die besagte Königin zu diesem Zeitpunkt trotz der unmittelbaren Nähe von 4 teilweise sehr drohnenreichen Völkern noch immer kein Begattungsmal hatte, und bei der Kürze der Einzelflüge eine ausreichende Besamung ohne sichtbares Zeichen sehr unwahrscheinlich ist, bleibt zu vermuten, daß sie noch wesentlich häufiger ausgeflogen wäre. Bis zum Abbautermin war erst eine Königin (Nr. 6) nachweislich, diese allerdings auf drei Flügen, begattet.

Verfliegen und Königinnenkampf. Eine Königin (Nr. 5) dieser Serie war nur 11 mal ausgeflogen. Sie verließ erst am 8. Lebenstag erstmalig den Stock, drang nach 13 minütigem Ausflug in das Nachbarkästchen ein, tötete kurzerhand die dort vorhandene Königin und verließ die nächsten 2 Tage den Stock nicht und flog am 11. Lebenstag auffällig wenig (s. Abb. 2b). Die abgestochene Königin wurde von ihrem Volk sofort eingeknäult, die Siegerin blieb unbehelligt, näherte sich später verschiedentlich der Toten und zeigte dabei Intentionsbewegungen zum Stechen. Am nächsten Mittag wurde die tote Königin herausgeschleppt und noch längere Zeit von einigen Arbeitsbienen auf dem Flugbrett betastet.

Zweite Inselserie. Entfernt stehende Drohnenvölker. Die nächste Serie begann unter veränderten Bedingungen. Die Drohnenvölker der Belegstelle “ Ost », auf der auch unsere Versuchsköniginnen standen, waren entfernt worden. Statt dessen hatte Drescher (Institut für Bienenkunde der Universität Bonn) auf der Belegstelle “ Loog " (Luftlinie $5 \mathrm{~km}$, s. Abb. 3) ein Volk mit über 1000 Drohnen zu Testzwecken aufgestellt. Das Königinnenmaterial stammte diesmal aus Bonn (geschlüpft : 24. 7.). Am 10. Lehenstag (2. August) wurden die EWK mit den neuen Königinnen eingesetzt. Bei Temperaturen um $22{ }^{\circ} \mathrm{C}$ unternahmen 2 der 6 Königinnen schon an diesem Tage einen Orientierungsflug. Der nächste Tag, windstill, Temperaturen bis $25^{\circ} \mathrm{C}$, lockte die Königinnen nur bis zu jeweils 12 Gesamtflugminuten bzw. bis 4 mal heraus, was bei dem günstigen Wetter in Anbetracht des Alters der Königinnen überraschend wenig ist. Die nächsten Tage brachten bei wechselhaftem Wetter mit Höchst- 
temperaturen zwischen 20 und $25^{\circ} \mathrm{C}$ noch eine Reihe kürzerer Ausflüge von maximal 3 Minuten Dauer. Insgesamt machten die Königinnen einen c lustlosen » Eindruck, d.h. ihre Flugaktivität war unerwartet gering und - soweit man auf Begattungsergebnisse wartete - schien sich die ganze Serie in Anbetracht der entfernt stehenden Drohnen nicht zu lohnen.

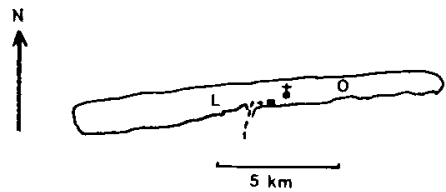

Авв. 3. - Die beiden Belegstellen auf der Insel Juist sind $5 \mathrm{~km}$ von einander entfornt. Die Versuchsköniginnen standen auf der Belegstelle « Ost 》 (O). Im letzten Versuch standen die Drohnen auf " Loog 》 (L).

FIG. 3. - Les deux stations de fécondation de l'île de Juist sont distantes de $5 \mathrm{~km}$. Pour les expériences les reines avaient ếe placées à la station "Ost» (O.). Durant la dernière expérience les mâles étaient à la station " Loog" (L.).

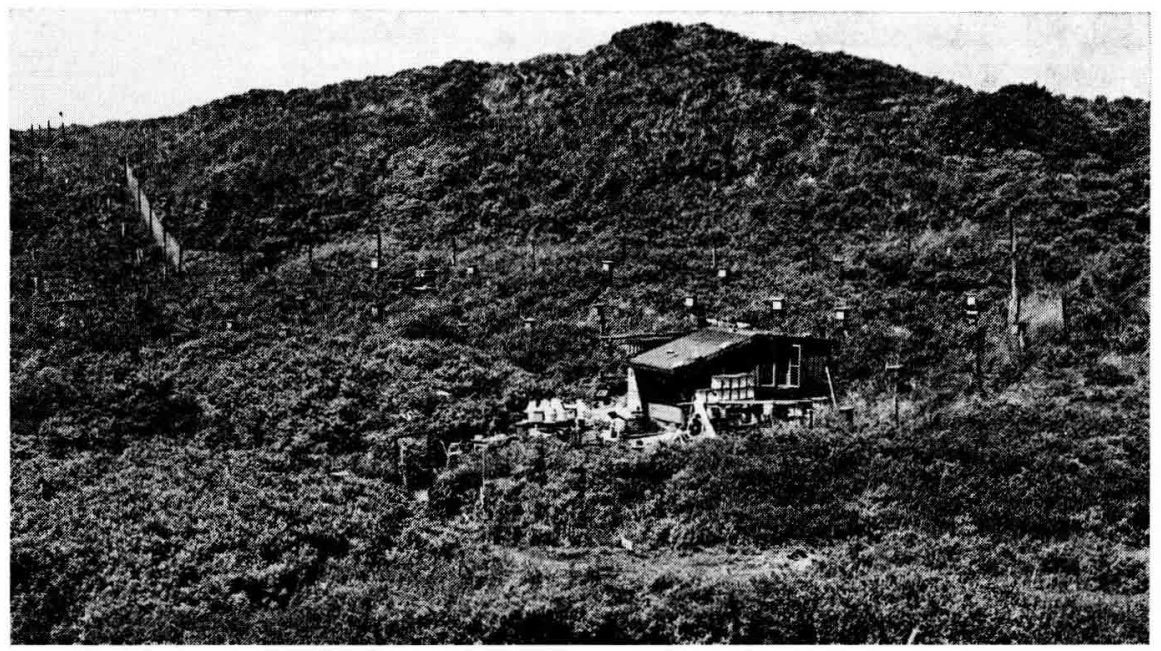

Авв. 4. - Die Belegstelle «Ost 》 befindet sich in einem nach Osten geöffneten Dünentalkessel. (Foto Gröl).

Fig. 4. - La station de fécondation « Ost » est située dans un cirque de duncs ouvert à l'est. (Photo Gröl).

Der 11. 8. (19. Lebenstag) war ein Tag mit wechselnder Bewölkung und mäßigem Wind, an dem die flugfreudigste Königin 4 mal ausflog, jedesmal kürzer als 1 Minute. Der 12. 8. war sehr windig, und ab Mittag herrschte 
strömender Regen. Trotzdem schickten sich 2 Königinnen an, auszufliegen, kehrten aber sofort wieder heim. Eine flog um 12.30 Uhr bei einer Außentemperatur von nur $16^{\circ} \mathrm{C}$ und einer Windgeschwindigkeit von $5 \mathrm{~m} / \mathrm{sec}$, in der geschützten Mulde der Belegstelle, dicht über der Strauchvegetation. Am 13. 8. (21. Lebenstag) flogen bei relativ windstillem und sonnigem Wetter mit Temperaturen bis zu $23^{\circ} \mathrm{C}$ die Königinnen nach kurzen Vorflügen für längere Zeit (bis zu 28 Minuten) aus. Fünf Königinnen kamen mit Begattungsmal zurück, eine davon 2 mal, eine mit mindestens einem Begattungsmal hatte ihr Markierungsplättchen verloren und konnte nicht mehr lückenlos überwacht werden. Der nächste Tag war bei höherer Luftfeuchte noch wärmer, bis $26^{\circ} \mathrm{C}$. Auch diesmal flogen nach mehreren kurzen Vorflügen die Königinnen für längere Zeit aus. Drei Königinnen trugen ein Begattungszeichen, eine zum ersten und eine zum dritten Mal. Am nächsten, zwar warmen (his $27^{\circ} \mathrm{C}$ ) aber ziemlich windigen Tage flogen 2 Königinnen ganz kurz aus. Am folgenden 24. Lebenstag ging die erste Königin in Eiablage, die anderen begannen am 25. Tag zu legen. Eine spätere Spermatozoenzählung durch Drescher ergab bei allen 5 Königinnen (eine war beim Einknäueln getötet worden) einen mittleren bis guten Spermienvorrat : 3,67 bis 7,81 Millionen (Mittel : 5,69 Millionen); die mit Abstand am spärlichsten besamte Königin hatte nur ein Begattungsmal.

\section{3.-3. Verhalten der Arbeiterinnen gegenüber der Königin}

Daß Arbeitsbienen oft aggressiv gegen Königinnen sind, ist jedem Praktiker zur Genüge bekannt. Nach meinen Beobachtungen scheint es mir fast berechtigt, Angriffe seitens der Bienen gegen junge von Hochzeitsflügen heimkehrende Königinnen als Regel zu bezeichnen. In vielen Fällen, bei allen Versuchsserien, hatte die Königin beim Heimflug erhebliche Schwierigkeiten, überhaupt zu landen, da sie von den Flugbrettbienen daran gehindert wurde. Manchmal gab es Angriffe aus dem Fluge, wobei die Königin öfters abstürzte, aber nie zu Schaden kam. Verschiedentlich, meist nach einer Begattung, wurden Königinnen stundenlang eingeknäult. Das war leicht zu beobachten, wenn man die Seitenwände der Schutzhäuschen entfernte. Da dies, um die Tiere nicht ständig zu stören, oft unterblieb, ist anzunehmen, daß etliche Knäuel übersehen wurden. Von 5 protokollierten Fällen endete einer für die Königin tödlich. Welche Faktoren für die Abwehr der Königin am Flugloch, wovon z.B. auch ENGLERT (1971) berichtet, wirksam sind, läßt sich bisher nicht sagen. Derartiges Verhalten wird bei heißem wie bei kühlem Wetter gezeigt, nach langen wie nach kurzen Ausflügen; Begattungserfolg oder -mißerfolg kann auch nicht ausschlaggebend sein, denn auch eine Königin mit Begattungsmal kann abgewehrt werden.

Dieser letzte Punkt widerspricht den Beobachtungen von Hammany 
(1957), denen zufolge die frisch begattete junge Königin erst am nächsten Tag wieder den vor der Begattung üblichen Angriffen ausgesetzt ist. Dieselbe Autorin und später unter speziellerer Fragestellung auch SzaBo und Townsend (1974) wiesen nach, daß das Alter der Bienen ein bedeutsamer Faktor im Verhalten gegenüber einer unbegatteten Königin ist. Nur wenige Tage alten Bienen fehlt die Aggressivität gegen unbegattete Königinnen. Damit entfällt - in Hammanss Experiment - aber gleichzeitig ein notwendiger Auslöser für den Königinnenausflug. Vor allem alte, größtenteils drohnenbrütige Bienen treiben dagegen die Königin besonders rasch zum Ausflug; sie fliegen - wie HammanN beobachtete - statt ihrer unter Umständen selbst schwarmartig aus. Normalerweise dürfte aber ein “ Hochzeitsschwarm » kaum je vorkommen. Bei meinen Beobachtungen - das Alter der Bienen war in diesen Versuchen nicht definiert - kam die Königin allein oder mit einzelnen Bienen oder auch mit großem Gefolge auf das Flugbrett. Nach dem Abflug reckten dann die stets zurückbleibenden Arbeiterinnen oft den Vorderkörper und die Vorderbeine empor, was ganz den Beobachtungen der obengenannten Autorin entspricht.

Bisweilen kam es vor, daß die Königin bei niedrigen Temperaturen eine sehr geringe Flugbereitschaft zeigte; sie versuchte, wieder in das Flugloch zurückzuschlüpfen, wurde daran jedoch von Bienen durch Anstoßen und Drängen gehindert. In solchen Fällen war ein feindseliger Empfang bei der Rückkehr mit höchster Wahrscheinlichkeit vorauszusagen. In anderen Fällen war ein solcher aber auch keineswegs auszuschließen.

\section{4. - DISKUSSION}

\section{4.-1. Zur Aggressivität der Arbeiterinnen}

Szabo (1974) konnte unter speziellen Testbedingungen nachweisen, daß auch das Alter der Königin und ihre Attraktivität im Zusammenhang mit der Aggressivität der Arbeitsbienen eine große Rolle spielen. Die Angriffe erreichten danach ein Maximum bei 21 Tagen. Unter den ( natürlichen ), schon durch das wechselnde Wetter uneinheitlichen Bedingungen des freien Ausfluges und bei der geringen Zahl der Versuchstiere ließ sich bei meinen Versuchen eine solche Abhängigkeit nicht klar aufzeigen.

Was in den beiden beobachteten Fällen tödlicher Aggressivität die Bienen veranlaßt haben könnte, ihre wiederholt begattete Königin zu töten (ảaß sie es taten, ist zumindest in einem Fall wahrscheinlich), läßt sich vorläufig genauso wenig klären, wie die Angriffe nach ihrer Rückkehr. Ein biologischer (arterhaltender) Sinn solcher Verhaltensweisen ist schwer zu finden, jedoch darf man nach einer möglichen Ursache suchen. Sie könnte darin liegen, daß die zum Ausfliegen der Königin offenbar unabdingbar notwendige Aggressi- 
vität der Arbeiterinnen bis zu ihrer Rückkehr infolge eines trägen, wie auch immer gearteten Motivationsmechanismus auf einen sehr hohen Pegel gestiegen ist, gleichgültig, ob die Königin begattet wurde oder nicht.

\section{4.-2. (Fernpaarungen )}

Relativ zur ersten Inselserie brachte die zweite mit entfernt stehenden Drohnen überraschend gute Begattungsergebnisse. Man kann schwer ausschließen, daß sie auf meteorologische Faktoren, genetisch bedingte Eigenarten oder andere Umstände (auch Zufall) zurückzuführen sind. Denkbar wäre aber immerhin, daß sich Königinnen und Drohnen allgemein in größerer Entfernung vom Stock paaren, was auch Befunde von Woyke bereits aus dem Jahre 1956 wahrscheinlich machen (Woyke 1962) : Königinnen, die $2,5 \mathrm{~km}$ entfernt vom nächsten Bienenstand aufgestellt waren, wurden in kürzerer Gesamtflugzeit deutlich besser begattet als solche, die in unmittelbarer Nachbarschaft von Drohnenvölkern standen. Beobachtungen von Begattungsmalen nach sehr kurzen Flügen beweisen allerdings, daß Belegung am Standort nicht ganz ausgeschlossen ist (KüNSTER und eigene Beobachtung). Bötтcher (1971) hatte auf einem Drohnensammelplatz, der fast nur von Cordovandrohnen beflogen war, nur wenige cd-begattete Königinnen erhalten. H. u. F. RutTnER (1971) kamen bei Versuchen mit verschiedenen genetisch markierten Drohnen zu gleichartigen Ergebnissen : nur 7,2\% der Königinnen wurden in unmittelbarer Nähe ihres Aufstellungsplatzes begattet, obgleich dort zahlreiche Drohnen vorhanden waren.

Populationsgenetisch äußerst günstig wäre ein Verhaltensmechanismus, der die Geschlechtspartner zur Paarung in der Regel weit vom Ausgangsvolk fortzöge. Besonders vorteilhaft wäre eine gegensinnige Flugrichtung der Drohnen und Königinnen, was durch unterschiedliche Reaktion auf einen beeinflussenden Faktor gewährleistet werden könnte. Aufgrund der leicht zu wiederholenden Beobachtung, daß am Faden hängende Königinnen von den Drohnen gegen den Wind angeflogen werden, stellen Bötrcher, MaUtz und WeIss (1975) die Frage, ob nicht die Königin auch natürlicherweise gegen den Wind fliegt, um den Drohnen eine bessere Möglichkeit ihrer Verfolgung zu geben. Wenn die Drohnen dagegen, solange sie keine Königin verfolgen, zunächst vorwiegend in Windrichtung fliegen - dafür geben die Inselbeobachtungen deutliche Hinweise - , dann wäre eine ständige genetische Durchmischung einer Bienenpopulation garantiert.

Falls sich ein solches Verhaltensmuster durch gezielte Experimente, die sich auf der Insel Juist mit ihren beiden Belegstellen nach Ende der Saison durchführen ließen, bestätigte, wäre eine Modifizierung der bisherigen Belegstellenpraxis dringend geraten, was auch schon von ENGLERT (1974) erwogen wird. 


\section{4.-3. Die besonderen Inselverhältnisse}

Ausflugshäufigkeit und Wind. In vieler Hinsicht weichen die Daten, die die Inselversuche lieferten, von den auf dem Festland ermittelten ab. Besonders fällt die Ausflugshäufigkeit auf. Ähnlich häufige und kurze Ausflüge auf Nordseeinseln, wenn auch nicht ganz so extrem, beobachteten auch Drescher, Englert und Künster. Drescher erklärt die Kürze der Flüge damit, daß die Königinnen, wenn sie die Höhe der Deichkrone erreicht haben, wegen der Windverhältnisse gleich wieder umkehren. ENGLERT (1971) vermutet, daß auf der Insel bei günstigem Wetter weniger oft geflogen würde; sie beobachtete außerdem, daß die nach Osten gerichteten Königinnen insgesamt häufiger ausfliegen als die nach Westen oricntierten. Sie führt das auf die unterschiedliche Besonnung des Flugbretts zurück. Man sollte in diesem Zusammenhang jedoch berücksichtigen, daß in unseren Breiten gewöhnlich Westwind herrscht und daß eine Königin, der der Wind schon ständig ins Flugloch bläst, wahrscheinlich auch deshalb seltener ausfliegen wird. 1974 berichtet ENGLERT auch von einem Beobachtungstag mit starkem Ostwind, an welchem 23 Südwestausflügen nur 4 Nordostausflüge gegenüberstanden. Es erscheint nur wenig zweifelhaft, daß von allen speziellen Inselfaktoren der Wind die größte Rolle spielt.

Flughöhe und Wind. Als Fluggeschwindigkeit der Sammlerinnen wird von verschiedenen Autoren 6-8 m/sec angegeben (Zit. nach ZaNDER-Weiss). Die schnellsten Flieger sollen $12-14 \mathrm{~m} / \mathrm{sec}$ erreichen. Während der Untersuchungen auf Juist herrschten bei gutem Drohnenflug oft Windgeschwindigkeiten zwischen 3 und $5 \mathrm{~m} / \mathrm{sec}$ in geschützter Mulde dicht über der Strauchvegetation; auf einer nahe gelegenen Düne etwa das Dreifache. Geschwindigkeiten von $14 \mathrm{~m} / \mathrm{sec}$ waren auf der Düne überhaupt keine Seltenheit, und in höheren Luftschichten ist mit noch größeren Windgeschwindigkeiten zu rechnen, die mit Sicherheit das Kompensationsvermögen der Bienen überfordern. An solchen windigen Tagen, und das sind auf den Inseln wohl die meisten, sieht man die Drohnen im windgeschützten Tiefflug gegen den Wind zum Stand zurückkommen, während sie sich offenbar zunächst in höheren Luftschichten abtreiben lassen.

Drohnensammelplätze. Vorausgesetzt, die Ostfriesischen Inseln böten mit ihren bis ca $20 \mathrm{~m}$ hohen Dünen genügend markante Horizontlinien für einen Drohnensammelplatz, die Windverhältnisse würden nur in Ausnahmefällen seine Benutzung erlauben. Die offensichtliche Windabhängigkeit der Drohnenflugrichtung spricht sehr dafür, daß Drohnensammelplätze, wenn auf Juist überhaupt vorhanden, hier eine wechselnde Rolle spielen. Im Binnenland werden die paarungsauslösenden Reize, die von der Königin ausgehen, erst ab einer bestimmten Flughöhe wirksam, wenigstens 6-15 m (PaIN und RutTNer 1963, zit. nach Zander-Weiss). Bei stärkerem Wind sinkt die 
Flughöhe der Drohnen, während sie bei heißem Wetter bis $50 \mathrm{~m}$ steigt; in Küstennähe beträgt die Flughöhe nur 2-5 m (Diskussion nach dem Referat von H. Ruttner 1972).

Neben den olfaktorischen spielen bekanntlich auch optische Reize eine Rolle (F. Ruttner 1957). Unter den Inselverhältnissen konnten wir in nur 2-3 m Höhe die meisten Drohnen zu kurzen Anflügen an ein am Faden flatterndes schwarzes Plastikplättchen veranlassen, und das in einem Dünental. 6-7 $\mathrm{m}$ war im allgemeinen schon viel zu hoch. Eine gekäfigte oder direkt an einen dünnen Stab gebundene Königin lockte wohl einen " Kometenschweif 》 an, weitergehende Paarungsversuche blieben aber aus, sie fanden hingegen statt in einer Höhe von nur etwa 1,50 m, wenn die Königin frei an einem Faden schwebte; natürlich ohne Erfolg, da die Stachelkammer nicht geöffnet war. Ein Drohn hielt sich 16 Sekunden an einer Königin fest; während dieser Zeit saß vorübergehend noch ein zweiter darauf. Auf der Düne herrschten während dieser Beobachtung Windgeschwindigkeiten von $10-15 \mathrm{~m} / \mathrm{sec}$.

Biologisch sinnvoll angepaßte Flughöhe bei der Paarung. Diese Versuche zeigen, daß die Drohnen mindestens unter bestimmten Umständen auf der Belegstelle selbst und in geringer Flughöhe begattungslustig sind. Etliche Zufallsbeobachtungen, die von Belegstellenleitern bestätigt werden, ergeben, daß auch freifliegende Königinnen auf der Insel in wenigen Metern Höhe von Drohnen verfolgt werden. Wenn der Drohn die ihren Flug beschleunigende Königin eingeholt hatte, stürzte nach unseren Beobachtungen das Paar jedesmal ab. Nur in einem dieser Fälle kam die Königin mit Begattungsmal zurück. Zwischen Absturz und Rückkehr lagen jedoch mehrere Minuten, die für einen erneuten Flug ausgereicht hätten. Wahrscheinlich benötigen die Bienen für eine erfolgreiche Paarung eine gewisse Fallhöhe, obgleich auch dann Abstürze häufig vorkommen dürften. Auf der Insel Vulcano fand sich öfters feiner Sand am Begattungsmal (Alber, Jordan, F. u. H. Ruttner 1955; F. Ruttner 1957). Dieser Notwendigkeit einer gewissen Fallhöhe wird das im Binnenland gezeigte Verhaltensmuster gerecht, dem zufolge sich ein Drohn erst ab einer gewissen Mindesthöhe für eine Königin interessiert. Das Verhalten der Drohnen scheint aber sinnvollerweise so plastisch zu sein, daß sie unter entsprechenden Windbedingungen den unteren Grenzwert extrem absenken können. Dadurch ist die Chance einer Begattung immer noch besser, als wenn sie jeden Versuch unterließen. Ein Fehlschlag bleibt ohne fatale Folgen, das ließ sich auf Juist zumindest für die Königin oft beobachten. RutTner (1957) berichtet auch von solcherart abgestürzten Drohnen, die ohne Zeichen einer Eversion wieder aufflogen. Katastrophale Folgen hätte es hingegen für beide Paarungspartner, wenn sie ohne Rücksicht auf dieWindverhältnisse die auf dem Festland üblichen Flughöhen ansteuern würden.

Eingegangen im Januar 1976.

Reçu pour publication en janvier 1976. 


\section{DANKSAGUNG}

Im Zusammenhang mit dieser Arbeit möchte ich zahlreichen Personen danken, besonders Prof. Dr. C. Ullrich für die Zusammenarbeit an diesem Projekt. Er machte auf das Inselproblem aufmerksam und sorgte an unserer Hochschule (PH Ruhr) für besonders gute Forschungsbedingungen. Er ermöglichte auch die wissenschaftliche Nutzung der Belegstelle Juist. Gedankt sei auch dem Belegstellenleiter Herrn Graubner, den Herren Gröl und Simbrey und nicht zuletzt meinem Bruder Rutger (Entwicklung der Elektronik) und Prof. Dr. W. Drescher (Zusammenarbeit bei der letzten Inselserie).

\section{RÉSUMÉ}

On a observé les vols de jeunes reines carnica à l'aide d'un dispositif de contrôle photoélectronique qui enregistre automatiquement l'instant où la sortie d'individus marqués déclenche automatiquement l'alarme (fig. 1). Les principales données figurent dans le tableau 1.

Sur le continent (Hagen, région de moyennes montagnes située entre la Ruhr et le Sauerland) chaque vol a duré en moyenne bien plus longtemps que sur l'île de Juist dans la Mer du Nord. Par contre le nombre de vols (comparer la fig. 2) sur le continent est bien plus réduit que sur l'île. On considère que la présence habituelle sur l'île d'un vent violent est la principale raison de ces vols courts et fréquents.

Les ouvrières se sont montrées souvent très agressives vis-à-vis de la reine qui revenait (même si elle s'était accouplée).

Tandis que sur le continent les tentatives d'accouplement n'ont lieu qu'à des altitudes assez élevées et de préférence sur les lieux de rassemblement de mâles, sur l'île de la Mer du Nord, aux dunes hautes d'environ $20 \mathrm{~m}$, les mâles ne volent qu'à quelques mètres d'altitude. A ces faibles altitudes aussi ont lieu des tentatives d'accouplement, qui, dans les cas observés, ont abouti à la chute sur le sol. Avec une reine captive les mâles n'ont pas tenté de s'accoupler à plus de 1,50 $\mathrm{m}$ de haut. On discute la signification biologique de ce comportement. On n'a pas pu mettre en évidence sur l'île de lieu de rassemblement de mâles.

Dans la première série d'expériences sur l'île, il y avait à proximité des colonies riches en mâles; le résultat des accouplements fut très mauvais. Dans la deuxième série les populations de mâles étaient éloignées de $5 \mathrm{~km}$. (L fig. 3); là le résultat des accouplements fut bon.

Il semble que les mâles se laissent avant tout pousser par le vent, tant qu'ils ne poursuivent pas de reine. Au cas où les reines voleraient principalement contre le vent, un accouplement dans les airs serait improbable. Ceci est significatif du point de vue biologique, mais laisserait apparaître comme mal approprié l'usage que l'on a fait jusqu'à présent des stations de fécondation (populations de mâles et de reines en un même endroit).

\section{LITERATUR}

Alber M., Jordan R., Ruttner F.u.H., (1955), Von der Paarung der Honigbiene. Zeitschrift für Bienenforschung 3, 1-18.

Böтtcher F. K., (1971), Wo paaren sich die Bienenköniginnen? XXIII internationaler Bienenzüchterkongreß, Moskau 443-445.

Böttcher F. K., Mautz D., Weiss K. (1975), Die Tätigkeit der Bayerischen Landesanstalt für Bienenzucht Erlangen im Jahre 1974. Der Imkerfreund 30, 75-85.

Büdel A. u. Henold E., Biene und Bienenzucht. Ehrenwirth, München, 1960. 
Drescher W., (1965), Untersuchungen zur Zuflugsicherheit der Inselbelegstelle Mellum. Zeitschrift für Bienenforschung 8, 49-54.

Englent E., (1971), Paarungsversuche auf der Insel Neuwerk. Allg. dtsche. Imkerztg. 5, 195199.

Englert E., (1972), Paarungsversuche auf der Insel Neuwerk. Apimondia, Internationales Symposium, Lunz, 1972, S. 49-52.

Englert E., (1974), Paarungsverhalten von Königinnen und Drohnen. Allg. dische Imkerztg., 8, 3-7.

Hammann E., (1957), Wodurch werden die unbegatteten Königinnen zum Ausflug veranlaßt? Insectes sociaux 4, 91-106.

Künster H., (1974), Kritische Bewertung der Belegstellen für Bienenköniginnen unter Berücksichtigung neuer Befunde ihrer Paarungsbiologie. Staatsexamensarbeit, Universität Bonn.

Meinen G., (1970), Wie sicher ist die Inselbelegstelle Spiekeroog vor fremdrassigem Drohneneinflug. Nordwestdeutsche Imkerzeitung 22, 128-130.

Meinen G., (1970), Unter welchen klimatischen Bedingungen finden auf den ostfriesischen Inselbelegstellen Paarungsflüge statt? Nordwestdeutsche Imkerzeitung 22, 207-209.

Rutrser F., (1957), Die Sexualfunktionen der Honigbienen im Dienste ihrer sozialen Gemeinschaft. Zeitschrift für vergleichende Physiologie 39, 577-600.

RutTNer H. u. F. (1971), Drohnensammelplätze und Paarungsdistanz. XXIII internationaler Bienenzüchterkongreß, Moskau, S. 445-447.

RutTNer H., (1972), Neue Versuche über die Flugbahnen der Drohnen in den Alpen. Internationales Symposium, Lunz, 1972, S. 39-4l.

Szabo T. J. u., Townsend G. F., (1974), Behavioural studies on queen introduction in the honeybee. 1. Effect of the age of workers (from a colony with a laying queen) on their behaviour towards an introduced virgin queen. Journal of Apicultural Research 13, 19-25.

SzaBo T. J., (1974), Behavioural studies on queen introduction in the honybee. 2. Effect of age and storage conditions of virgin queens on their attractiveness to workers. Journal of Apicultural Research 13, 127-135.

Szabo T. J., (1974), Behavioural studies on queen introduction in the honeybee. 3. Relationship between queen attractiveness to workers and worker aggressiveness towards a queen. Journal of Apicultural Research 13, 161-171.

TRJAsкo W. W., (1956), Wiederholte Begattungsflüge und Mehrfachpaarung der Bienenkönigin, Leipziger Bienenzeitung 1956, 70, 173-174 (Übersetzung).

Verbeek B. u. R., (1974), Eine Methode zur Registrierung des Aus-und Einfliegens einzelner Bienen. Apidologie, 5, 289-293.

Woyke J., (1962), Natural and Artificial Insemination of Queen Honeybees. Bee World 43, 2125.

WoYke J., (1964), Causes of repeated Mating Flights by Queen Honeybees. Journal of Apicultural Research 3, 17-23.

WoYKe J., (1966), Wovon hängt die Zahl der Spermien in der Samenblase der auf natürlichem Wege begatteten Königin ab? Zeitschrift für Bienenforschung 8, 236-247.

Zander-WeIss, Das Leben der Biene. Verlag Eugen Ulmer, Stuttgart, 1964. 